\title{
Calcium Phosphate Mineralization beneath a Polycationic Monolayer at the Air-Water Interface
}

\author{
Mathias Junginger, Katarzyna Kita-Tokarczyk, Thomas Schuster, \\ Jürgen Reiche, Felix Schacher, Axel H. E. Müller, Helmut Cölfen, \\ Andreas Taubert*
}

The self-assembly of the amphiphilic block copolymer poly( $n$-butyl methacrylate)-blockpoly[2-(dimethylamino)ethyl methacrylate] at the air-water interface has been investigated at different pH values. Similar to Rehfeldt et al. (J. Phys. Chem. B 2006, 110, 9171), the subphase $\mathrm{pH}$ strongly affects the monolayer properties. The formation of calcium phosphate beneath the monolayer can be tuned by the subphase $\mathrm{pH}$ and hence the monolayer charge. After $12 \mathrm{~h}$ of mineralization at $\mathrm{pH} 5$, the polymer monolayers are still transparent, but transmission electron microscopy (TEM) shows that very thin calcium phosphate fibers form, which aggregate into cotton ball-like features with diameters of 20 to $50 \mathrm{~nm}$. In contrast, after $12 \mathrm{~h}$ of mineralization at $\mathrm{pH} 8$, the polymer film is very slightly turbid and TEM shows dense aggregates with sizes between 200 and $700 \mathrm{~nm}$. The formation of calcium phosphate is further confirmed by Raman and energy dispersive X-ray spectroscopy. The calcium phosphate architectures can be assigned to the monolayer charge, which is high at low $\mathrm{pH}$ and low at high $\mathrm{pH}$. The study demonstrates that the effects of polycations should not be ignored if attempting to understand the colloid chemistry of biomimetic mineralization. It also shows that basic block copolymers are useful complementary systems to the much more commonly studied acidic block copolymer templates.

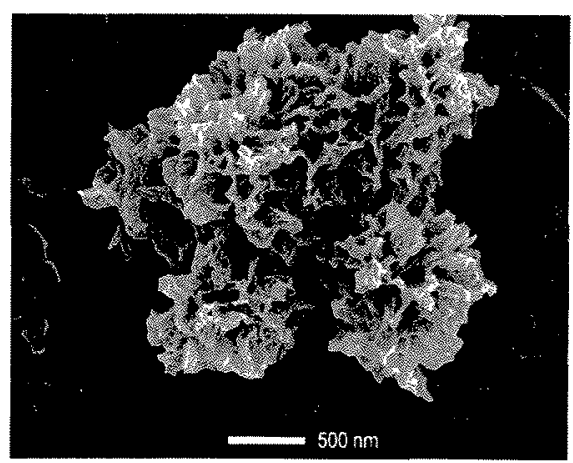

M. Junginger, A. Taubert

University of Potsdam, Institute of Chemistry, 14476 Potsdam, Germany

E-mail: ataubert@uni-potsdam.de

M. Junginger, H. Cölfen, A. Taubert

Max Planck Institute of Colloids and Interfaces, 14476 Potsdam,

Germany

H. Cölfen

Current address: University of Konstanz, Physical Chemistry,

78457 Konstanz, Germany

K. Kita-Tokarczyk, T. Schuster

Department of Chemistry, University of Basel, 4056 Basel,

Switzerland
K. Kita-Tokarczyk

Current address: Centre for Molecular Nanoscience, School of Chemistry, University of Leeds, Leeds LS2 gJT, UK

J. Reiche

University of Potsdam, Institute of Physics and Astronomy, 14476 Potsdam, Germany

F. Schacher, A. H. E. Mülle

Makromolekulare Chemie II, University of Bayreuth,

Naturwissenschaften II (NW II), 95440 Bayreuth, Germany

F. Schacher

Current address: School of Chemistry, University of Bristol, Bristol BS8 1TS, UK 


\section{Introduction}

Organic/inorganic or polymer/inorganic hybrid materials are of great interest for a variety of applications, e.g., chromatography or implants. Many of the newer synthetic developments have been inspired by nature. This is because countless organisms synthesize highly organized and specialized materials from organic scaffolds and chemically rather simple inorganic compounds like calcium carbonate or calcium phosphate. ${ }^{[1,2]}$ Nature makes most of these highly advanced hybrid materials at roughly ambient conditions and chemists have tried to mimic these processes for the synthesis of novel functional materials. ${ }^{[3-7]}$

In order to rationally design bioinspired composite materials, it is necessary to understand and quantify the molecular and supramolecular parameters that control the formation, structure, and properties of a given organic/ inorganic combination. As a consequence, there have been many studies, for example, on the mineralization of calcium carbonate, which is one of the most important biominerals. ${ }^{[7-15]}$

Calcium phosphate is also an important biomineral, but it has been studied much less. ${ }^{[4]}$ In particular, there have been only a few studies on the mineralization of polymeric surfaces with calcium phosphate. This is intriguing, because in nature, mineralization occurs in gel phases ${ }^{[16,17]}$ or at interfaces. ${ }^{[16]}$ Ngankam et al. have shown that calcium phosphate nucleation is dramatically reduced if multilayers of poly(ethyleneimine) (PEI), poly(sodium-4styrenesulfonate) (PSS), and poly(allylamine hydrochloride) (PAH) are deposited on a silica surface. ${ }^{[18]}$ The authors show that the surface charge (positive or negative) is of minor importance for the nucleation efficiency. The charge is important, however, in the crystal phase selection. This is similar to results obtained for $\mathrm{CaCO}_{3}$ nucleated under calixarene monolayers. ${ }^{[19]}$ Subsequently, Ball et al. have shown that poly(amino acid) multilayers of poly(L-glutamic acid) or poly(l-aspartic acid) and poly(l-lysine) efficiently control the lag times before nucleation. ${ }^{[20]}$ To our best knowledge, however, there is at the moment no explanation for this behavior. Spoerke et al. have shown that polyelectrolyte coatings are also viable for the modification of titanium surfaces. ${ }^{[21]}$ More recently, Schweizer et al, have shown that polyelectrolyte multilayers modified with calcium phosphate could be interesting coatings for cardiovascular stents. ${ }^{[22]}$

Besides the examples above, which were all synthesized using the layer-by-layer (LbL) approach, only a few examples of calcium phosphate mineralization at interfaces have been published. Walsh et al. have synthesized free-standing calcium phosphate films with adjustable porosities and morphologies. ${ }^{[23]}$ Zhang et al. have shown that monolayers of low-molecular-weight compounds are efficient templates for interfacial calcium phosphate mineralization control. ${ }^{[24]}$ More recently, Casse et al. have shown that monolayers of amphiphilic block copolymers at the air-water interface are useful model systems to study calcium phosphate mineralization. ${ }^{[25]}$ The advantage of this approach is that parameters such as $\mathrm{pH}$, ionic strength, chemical composition of the subphase, monolayer composition, etc. can be investigated independently. It is thus possible to develop an understanding of the role of different parameters on mineral film formation at a well-defined surface. The study shows that small supersaturations, $\mathrm{pH}$ values higher than $\sim 8$, and mechanical agitation of the subphase favor the formation of well-controlled, uniformly mineralized polymer monolayers. This is interesting because these are roughly (with the exception of the $\mathrm{pH}$ ) the conditions at which mineralization in nature occurs.

While most studies focus on the role of polyanions, several publications show that polycations are also efficient growth modifiers for calcium phosphate. ${ }^{[26-28]}$ These reports demonstrate that polycations in some cases efficiently stabilize calcium phosphate nanoparticles and strongly interact with various crystal phases. The current study evaluates the potential of amphiphilic block copolymer monolayers with a cationic block for calcium phosphate mineralization at the air-water interface. The major goal of this study is to provide a model system, which is comparable to the earlier study by Casse et al., ${ }^{[25]}$ yet enables the investigation of polycationic surfaces on calcium phosphate mineralization in much more detail than previously achieved. ${ }^{[18,20-22]}$

\section{Experimental Part}

\section{Chemicals}

Monosodium phosphate, calcium chloride trihydrate, tris(hydroxymethyl)aminomethane (Sigma-Aldrich), sodium acetate, hydrochloric acid, chloroform (spectroscopic grade) (Merck), and acetic acid (Roth) were used as received. All glassware was treated with a $\mathrm{H}_{2} \mathrm{O}_{2} / \mathrm{H}_{2} \mathrm{SO}_{4}(1: 2)$ mixture and rinsed with double distilled water. Water for Langmuir-Blodgett experiments was either double distilled from a dedicated quartz glass distillation setup or from a Millipore Millio with a resistivity of $18.2 \mathrm{M} \Omega \cdot \mathrm{cm}$.

Tetrahydrofuran (THF) for the anionic polymerization was distilled over $\mathrm{CaH}_{2}$ and $\mathrm{K}$ and was directly transferred to the polymerization reactor afterwards. Prior to the polymerization, the THF was cooled to $-20^{\circ} \mathrm{C}$ and $1 \mathrm{~mL}$ of sec-BuLi per $100 \mathrm{~mL}$ THF were added to the THF. The resulting yellow mixture was stirred for $15 \mathrm{~min}$ at $-20^{\circ} \mathrm{C}$ and then warmed to room temperature. The secBuLi reacted with the THF, to generate lithium alkoxides present during the polymerization. $N$-Butyl methacrylate (nBMA) and $N, N$ dimethylaminoethyl methacrylate (DMAEMA) were degassed by three consecutive freeze-thaw cycles. Tri( $n$-octyl)aluminum (Aldrich, 25\% solution in hexane) was then added until a yellow color persisted (typically $2 \mathrm{~mL}$ per $10 \mathrm{~mL}$ of monomer) and the 
resulting mixture was stirred under strict exclusion of light for $45 \mathrm{~min}$. Afterwards, the desired amount of monomer was condensed on a high vacuum line into either an ampoule or a Schlenck flask. 1,1-Diphenylethylene (DPE) was treated with secBuLi, distilled under reduced pressure and stored under a protective gas atmosphere.

\section{Silicon Wafers}

Silicon wafers were cleaned with a mixture of hydrogen peroxide and sulfuric acid (1:2) for $10 \mathrm{~min}$. Afterwards the wafers were rinsed thoroughly with deionized water and dried in a desiccator. Silicon wafers were rendered hydrophobic by evaporation deposition of bis(trimethylsilyl)amine (hexamethyldisilazane, HMDS). To that end, the silicon wafers and an open vial of HMDS were stored in a closed beaker for $24 \mathrm{~h}$ to allow the HMDS to evaporate and react with the surface silicon dioxide of the wafers.

\section{Synthesis of Poly(n-butylmethacrylate)-block-Poly[2- (dimethylamino)ethyl methacrylate] (PnBMA ${ }_{75}$-PDMAEMA ${ }_{147}$ ) P1}

To $500 \mathrm{~mL}$ of THF in a stirred tank reactor $0.2 \mathrm{~mL}$ of DPE $(0.198 \mathrm{~g}$, $1.1 \mathrm{mmol}$ ) and $0.71 \mathrm{~mL}$ of sec-BuLi $(1 \mathrm{mmol})$ were added at $-78^{\circ} \mathrm{C}$ to form the initiating system, 1,1-diphenylhexyllithium (DPHLi). Afterwards, $10.1 \mathrm{~mL}$ of $n \mathrm{BMA}(9 \mathrm{~g}, 63.3 \mathrm{mmol})$ were added with a syringe. The mixture was then slowly warmed to $-50^{\circ} \mathrm{C}$ and held at that temperature for $1 \mathrm{~h}$. DMAEMA $(22.6 \mathrm{~mL}, 21 \mathrm{~g}, 133.7 \mathrm{mmol})$ was then added with a syringe and polymerized at the same temperature for another hour. The reaction was quenched by the addition of $2 \mathrm{~mL}$ of degassed isopropyl alcohol. The polymer was purified by dialysis (MWCO $8000 \mathrm{~g} \cdot \mathrm{mol}^{1}$ ) against dioxane and dioxane/water $80 / 20$ mixtures to remove the $\mathrm{Li}^{+}$and freeze-dried afterwards. Characterization according to ${ }^{1} \mathrm{H}$ NMR spectroscopy and size exclusion chromatography (SEC) gave a molecular weight of $10600 \mathrm{~g} \cdot \mathrm{mol}^{-1}$ for the PnBMA block and $23100 \mathrm{~g} \cdot \mathrm{mol}^{-1}$ for the PDMAEMA block. The polymer is denoted as PnBMA ${ }_{75} \mathrm{PD}$ MAEMA $_{147}$ (subscripts denoting the respective degrees of polymerization) with an overall molecular weight of $33700 \mathrm{~g} \cdot \mathrm{mol}^{-1}$ and a polydispersity index (PDI) of 1.10.

\section{Langmuir Monolayer Experiments}

Surface pressure-area $(\pi-A)$ isotherms of P1 were recorded on a custom made Langmuir trough (KSV Finland) with a total area of $440 \mathrm{~cm}^{2}$ and two moveable barriers. Additional experiments were made on a Nima Technology (UK) 611 medium deposition trough with two moveable barriers. The stability measurements were performed on the Nima Technology trough. The troughs were placed on anti-vibration tables in a dust free room. All experiments were performed at room temperature.

Monolayers were prepared by spreading aliquots of 40 to $150 \mu \mathrm{L}$ of $\mathbf{P 1}$ solution (1.1 mg $\mathrm{mL}^{-1}$ ) in $\mathrm{CHCl}_{3}$ on pure water or aqueous solutions of $\mathrm{pH} 2,5$, and 8 with monosodium phosphate as additive. After spreading, a minimum of $10 \mathrm{~min}$ was allowed for the solvent to evaporate. The surface pressure $(\pi)$ was recorded to $\pm 0.1 \mathrm{mN} \cdot \mathrm{m}^{-1}$ with a Wilhelmy plate (chromatography paper, ashless Whatman Chr1) connected to an electrobalance.

Film stability was measured at different subphase $\mathrm{pH}$ values and during mineralization at different $\mathrm{pH}$ values. For stability measurements, films were compressed to $30 \mathrm{mN} \cdot \mathrm{m}^{-1}$ and the change of the mean molecular area was monitored for $16 \mathrm{~h}$ at room temperature.

Films were transferred at $30 \mathrm{mN} \cdot \mathrm{m}^{-1}$ and $0.5 \mathrm{~mm} \cdot \mathrm{min}^{-1}$ onto hydrophilic and hydrophobic silicon wafers modified with hexamethylsiliazane by Langmuir-Blodgett transfer. The transfer ratios were between 0.8 and 0.9 for successful transfers and 0.1 to 0.2 for unsuccessful transfers. The hydrophilic substrates were coated with a monolayer by placing the substrate in the subphase prior to spreading the polymer. The hydrophobic substrates were coated with a bilayer by a dipper downstroke-upstroke transfer. Films were dried at ambient conditions overnight before further experiments were done.

\section{Mineralization}

Mineralization experiments were conducted on both troughs at $30 \mathrm{mN} \cdot \mathrm{m}^{-1}$. For mineralization, polymers were spread from chloroform $\left(1.1 \mathrm{mg} \cdot \mathrm{ml}^{-1}\right)$ on a $2 \times 10^{-3} \mathrm{M} \mathrm{NaH}_{2} \mathrm{PO}_{4}$ aqueous subphase. The solutions were buffered with an acetate ( $\mathrm{pH} 5$ ) or a Tris ( $\mathrm{pH}$ 8) buffer. After chloroform evaporation, a $\mathrm{CaCl}_{2}$ solution was injected into the subphase using a Hamilton syringe. The subphase was continuously stirred by placing a small stirring rod in the dipping wells of the troughs.

\section{Atomic Force Microscopy (AFM)}

AFM measurements were done on a Molecular Imaging PicoLE (Agilent) with a multi-purpose scanner and a Solver Pro (NT-MDT, Russia).Images were acquired using a silicon cantilever type-NCHR pointprobe-plus (force constant $42 \mathrm{~N} \cdot \mathrm{m}^{2}$, NanoAndMore $\mathrm{GmbH}$ Wetzlar, Germany) in non-contact mode and a silicon cantilever AC160TS (force constant $42 \mathrm{~N} \cdot \mathrm{m}^{-1}$, Olympus) in tapping mode. All experiments were conducted in air at room temperature

\section{Electron Microscopy}

Transmission electron microscopy (TEM) was done on an FEI Morgani 268D with a tungsten source operated at $80 \mathrm{kV}$. Films were directly transferred onto copper TEM grids from the trough and imaged after overnight drying. Scanning electron microscopy (SEM) was done on a Philips XL30 FEG ESEM operated at $10 \mathrm{kV}$. Samples were sputtered with Pt prior to imaging. Energy dispersive $X$-ray spectroscopy (EDXS) experiments were performed with an EDAX spectrometer from 0 to $10 \mathrm{keV}$.

\section{Spectroscopy}

Transmission infrared (IR) spectra were recorded on a Thermo Nicolet Nexus 670 FTIR spectrometer. Spectra were recorded at room temperature from 400 to $4000 \mathrm{~cm}^{-3}$ with a resolution of 
$4 \mathrm{~cm}^{-1}$. Silicon wafers coated with mineralized monolayers were measured without further reconditioning. The silicon wafers were mounted on the sample holder and placed in the laser beam of the FT-IR spectrometer. Raman spectra were acquired on a CRM200 confocal Raman microscope (WITEC, Germany) with a linearly polarized excitation laser (diode pumped green laser, $\lambda=532 \mathrm{~nm}$, CrystaLaser) on the AFM samples.

\section{Results and Discussion}

\section{Polymer Monolayer Properties}

Figure 1 shows surface pressure-area $(\pi-A)$ isotherms of $\mathbf{P 1}$ at different $\mathrm{pH}$ values with and without $\mathrm{NaH}_{2} \mathrm{PO}_{4}$ in the subphase. At $\mathrm{pH} 2$, the $\pi-A$ isotherm shows only one transition, from gaseous to a liquid expanded phase. The lift-off area is about $2800 \AA^{2}$ and the collapse pressure of the monolayer is ca. $60 \mathrm{mN} \cdot \mathrm{m}^{-1}$.

At $\mathrm{pH} 5$, a quasi-plateau appears at $15 \mathrm{mN} \cdot \mathrm{m}^{-1}$ and $7000 \AA^{2}$. Up to a mean molecular area of $2500 \AA^{2}$ the surface pressure of this plateau increases only slightly. With further compression, the monolayer behaves like the isotherm at $\mathrm{pH} 2$, showing a collapse pressure at ca. $60 \mathrm{mN} \cdot \mathrm{m}^{-1}$. If $\mathrm{NaH}_{2} \mathrm{PO}_{4}$ is added to the subphase, the plateau (this time below $10 \mathrm{mN} \cdot \mathrm{m}^{-1}$ ) reaches from 8500 to $2000 \AA^{2}$ but the increase in surface pressure is less pronounced than at $\mathrm{pH} 5$. This isotherm resembles the isotherm observed at $\mathrm{pH} 2$ without phosphate. The lift-off area is around $12300 \AA^{2}$.

At $\mathrm{pH} 8$, the $\pi-A$ isotherms show a pronounced plateau between 3000 and $1500 \AA^{2}$. Below $1500 \AA^{2}$, the surface pressure again rapidly increases. The lift-off area is around $9500 \AA^{2}$. The $\pi-A$ isotherms of monolayers at $\mathrm{pH} 8$ without $\mathrm{H}_{2} \mathrm{PO}_{4}^{-}$and on pure water $(\mathrm{pH} \sim 7)$ are virtually identical to the isotherm just described for $\mathrm{pH} 8$ and with $\mathrm{H}_{2} \mathrm{PO}_{4}^{-}$in the subphase.

Qualitative long term film stability measurements during mineralization show that the films are not

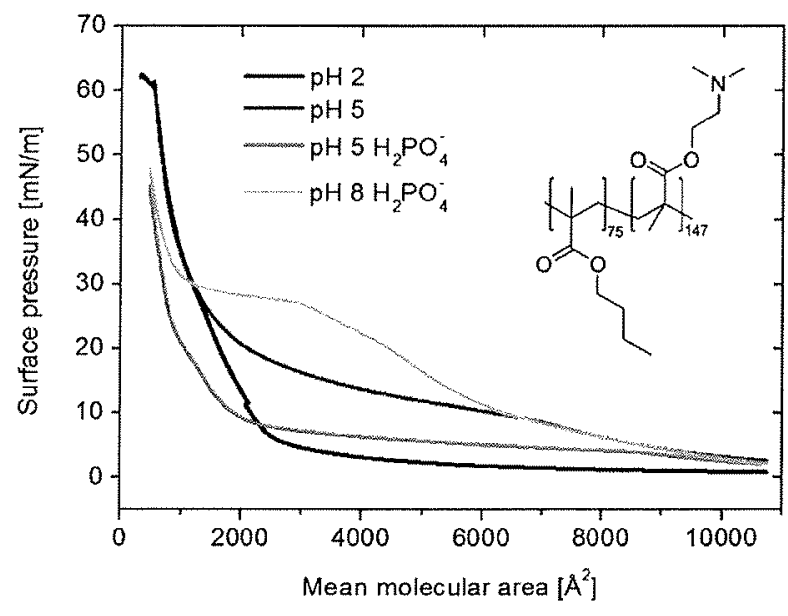

Figure 1. $\pi-A$ isotherms of $\mathbf{P 1}$ on different subphases. perfectly stable (data not shown). This is indicated by a decreasing mean molecular area versus time, suggesting that some of the polymer dissolves into the subphase. This dissolution is more pronounced at $\mathrm{pH} 2$, where ca. $100 \%$ of the amine groups of the polymer are charged. ${ }^{[29]}$

Figure 2 shows AFMimages of non-mineralized $\mathbf{P} \mathbf{1}$ and $\mathbf{P} \mathbf{1}$ mineralized at $\mathrm{pH} 5$ and $30 \mathrm{mN} \cdot \mathrm{m}^{-1}$ for $12 \mathrm{~h}$. The films were transferred onto hydrophobic and hydrophilic silicon wafers. On hydrophilic surfaces, the pure polymer monolayers are uniform and smooth with a root mean square (rms) surface roughness of $0.18 \mathrm{~nm}$ (Figure $2 \mathrm{a}$ ). In contrast to the hydrophobic substrates, it was, however, not possible to transfer the mineralized films, so a comparison with the films transferred onto hydrophobic substrates is not possible here.

On hydrophobic silicon, P1 exhibits a strong tendency towards dewetting with a feature height of the dewetted polymer up to ca. $100 \mathrm{~nm}$ (Figure 2b). Unlike on the hydrophilic surfaces, the mineralized films remain stable on the hydrophobic surfaces. The mineralized films are very smooth with a rms roughness of $2.19 \mathrm{~nm}$ and dot-like features with diameters between 20 and $50 \mathrm{~nm}$ (Figures $2 \mathrm{c}$ and d). For thickness measurements the mineralized film was scratched with a needle (Figure $2 \mathrm{c}$ ). However, these measurements have remained somewhat inconclusive, because it could not be assured that the lower part of the scratch is the actual substrate surface. The roughness of the surface in the scratch is very similar to the roughness on the mineralized film, and we thus suspect that some material remains in the scratch.

\section{Mineralization of the Polymer Monolayers}

Figure 3 shows TEM and SEM images of monolayers mineralized at $\mathrm{pH} 5$ and 8 at $30 \mathrm{mN} \cdot \mathrm{m}^{-1}$. TEM shows that after ca. $12 \mathrm{~h}$ at pH 5 small and uniform particles with an average diameter of $27 \pm 5 \mathrm{~nm}$ form. The particles are randomly arranged in two dimensions and there is no evidence of multilayer formation of these features. Higher magnification images reveal that numerous small filaments exist between the dark patches of the particles. The diameter of the filaments is between 2 and $3 \mathrm{~nm}$ and appears very uniform.

The particles formed at $\mathrm{pH} 8$ are much larger than those observed at $\mathrm{pH} 5$. They appear randomly at the monolayer, are irregular in shape, and have a large size distribution with dimensions between 300 and $3000 \mathrm{~nm}$. Higher magnification images show that these particles are composed of 100 to $600 \mathrm{~nm}$ long, elongated features. These features are in turn composed of numerous, 2 to $5 \mathrm{~nm}$ thin, roughly parallel fibrillar entities. In contrast to the filaments obtained at $\mathrm{pH}$ 5, these fibrillar entities are approximately parallel to one another (within one of 
a)

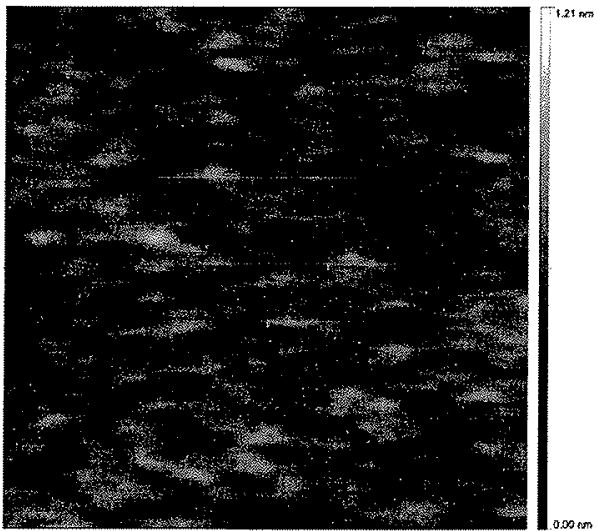

c)

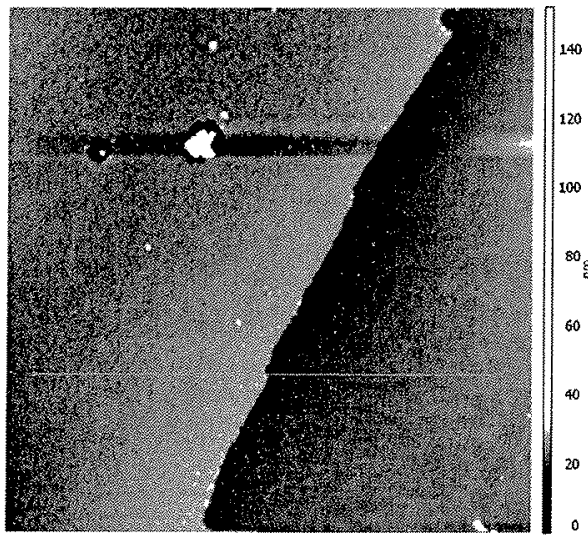

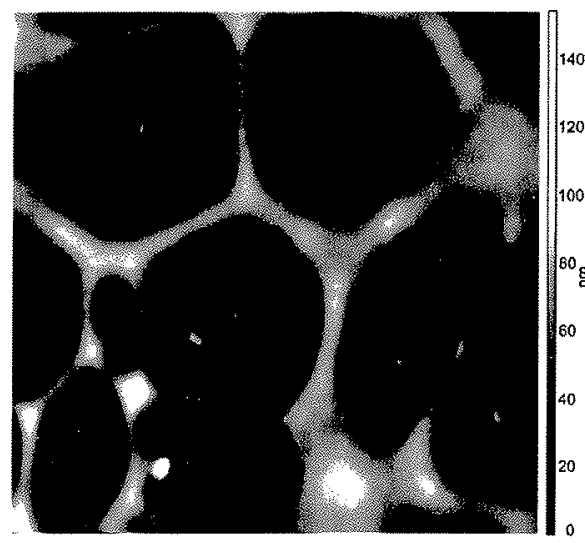

d)

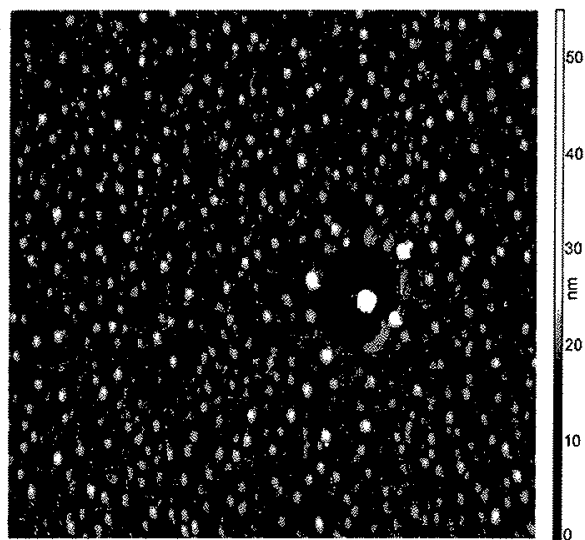

Figure 2. AFM images of P1 transferred on silicon wafers. a) P1 on hydrophilic silicon transferred by upstroke. b) P1 on hydrophobic silicon transferred by downstroke-upstroke. c,d) Two layers of mineralized P1 on hydrophobic silicon transferred by downstroke-upstroke at different magnifications. Transfer of mineralized films on hydrophilic substrates failed. AFM image sizes are a) $0.3 \times 0.3 \mu{ }^{2}$, $b, c) 10 \times 10 \mu \mathrm{m}^{2}$, and d) $3 \times 3 \mu \mathrm{m}^{2}$. Panel (a) is shown at a higher magnification than $(b, c)$ because there are no visible features at magnifications comparable to those of images shown in (b) and (c).

the elongated features) while the filaments obtained at pH 5 are randomly oriented.

SEM further shows that the large aggregates obtained at $\mathrm{pH} 8$ are quite rigid sponge-like materials, similar to earlier work at physiological conditions at $\mathrm{pH}$ 7.4. ${ }^{[30-32]}$ The EDX spectra show signals of calcium and phosphorus, which confirms the formation of a calcium phosphate mineral phase. The oxygen signal is attributable to both the mineral and the polymeric matrix, and the carbon signal arises from the polymer and the SEM sample support. Because of the sensitivity of the samples it was not possible to obtain electron diffraction patterns or $X$-ray diffraction data to definitely assign the crystal phase. This observation is consistent with previous studies. ${ }^{[25,33,34]}$

It has been shown earlier that stirring the subphase is a critical point and that homogeneous films only form if the subphase is mechanically agitated. ${ }^{[25]}$ Similarly, Figure 4 shows that without stirring, large calcium phosphate crystals form during the mineralization at the P1 monolayer at $\mathrm{pH} 5$ at $30 \mathrm{mN} \cdot \mathrm{m}^{-1}$. Unlike in the stirred subphases discussed so far, a more complex film with two distinct features forms. First, there is a background film. It resembles the thin filaments described above, although it appears to be denser. Second, there are large, dark features with a crystal-like shape. Interestingly, these larger features seem to exhibit some correlation in the sense that many of the edges of neighboring particles are roughly parallel. One possible explanation can be found in the transfer process after the mineralization because the removal of the substrate from the interface during the transfer is known to induce order in transferred films.

The assignment of the calcium phosphate crystal phase grown on the monolayers is not straightforward. This is because $\mathrm{X}$-ray diffraction does not yield enough counts and electron diffraction destroys the films within a second. Moreover, IR spectra (data not shown) of the mineralized films have a very poor signal to noise ratio. The spectra only 

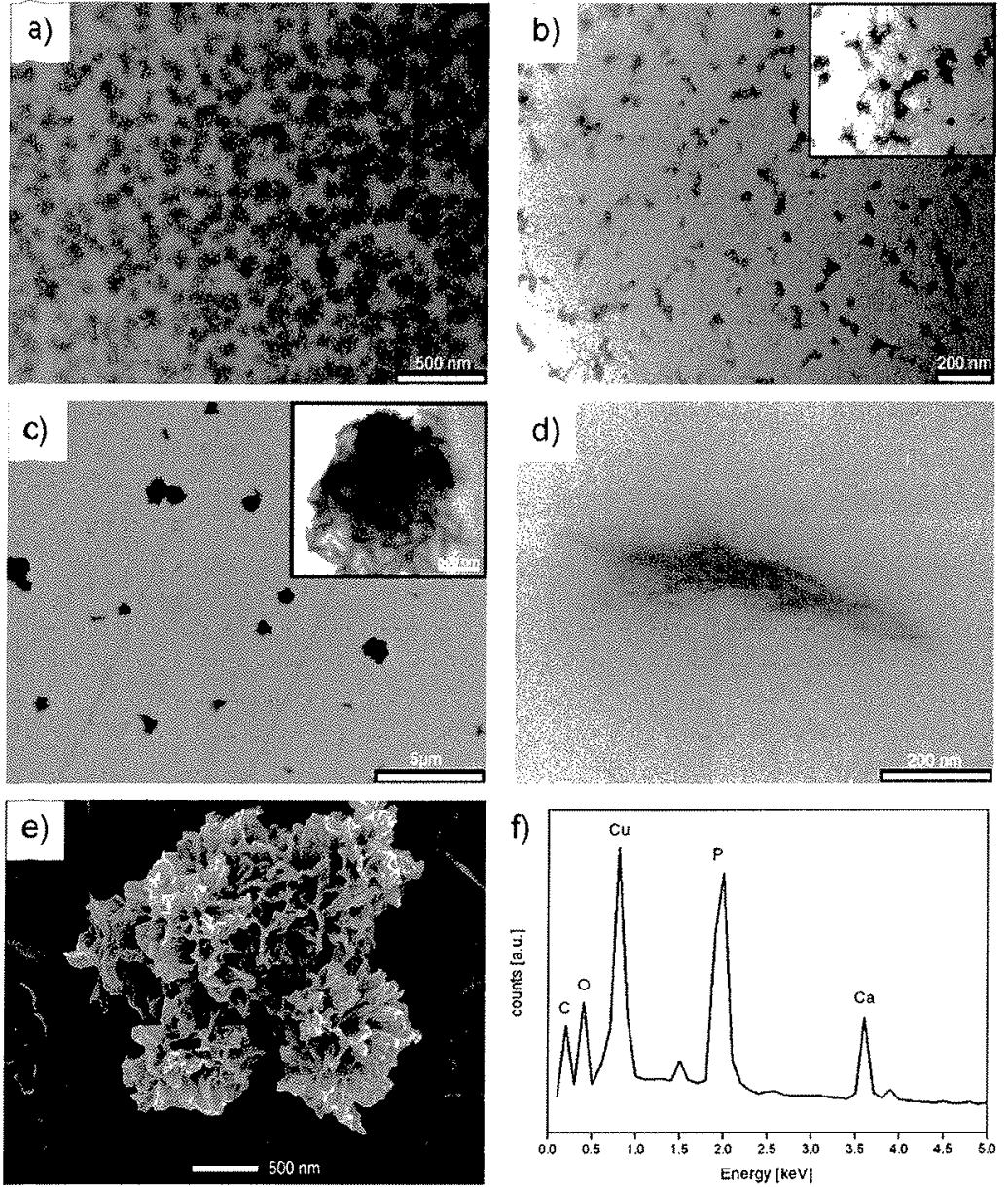

Figure 3. TEM (a-d) and SEM (e) images of mineralized monolayers after $12 \mathrm{~h} . \mathrm{a}, \mathrm{b}$ ) Monolayer mineralized at $\mathrm{pH}$ 5. $\mathrm{c-e}$ ) Monolayer mineralized at $\mathrm{pH} 8$. Highlighted area in (b) was contrast-adjusted to better show the filaments mentioned in the text. Inset in (c) is a magnification of a large particle formed at $\mathrm{pH} 8$. Panel (d) shows one of the elongated features (a component of the large particles) composed of roughly parallel fibrillar entities. See text for details. f) EDX spectrum of a mineralized aggregate obtained at $\mathrm{pH} 8$.

show two broad humps between 3700 and $2000 \mathrm{~cm}^{-1}$ and between 1789 and $1724 \mathrm{~cm}^{-1}$ along with a broad intense band at $1079 \mathrm{~cm}^{-1}$. The first signal is probably attributable to overlapping water, $\mathrm{NH}$, and $\mathrm{CH}$ vibrations, although the signal is too non-descript for a clear assignment. The bands at around $1700 \mathrm{~cm}^{-1}$ can be assigned to the ester carbonyl bonds of the polymer and the band at $1079 \mathrm{~cm}^{-1}$ is attributed to the ester $\mathrm{C}-\mathrm{O}$ vibration of the polymer or to the oxidized silicon support.

Raman spectra of samples grown at $\mathrm{pH} 5$ only show two bands at 520 and $996 \mathrm{~cm}^{-1}$, which can be assigned to the Si substrate. In contrast, Figure 5 shows a representative Raman spectrum of a film mineralized at $\mathrm{pH}$. The bands at $421,598,955$, and $1061 \mathrm{~cm}^{-1}$ can be assigned to $v_{2}, v_{4}, v_{1}$ and $\nu_{3}$ stretching vibrations of hydroxyapatite (HAP), respectively. A sharp signal at $519 \mathrm{~cm}^{-1}$ can be assigned to the $\mathrm{Si}-\mathrm{O}$ stretching vibration, $^{[35]}$ and a broad hump at $769 \mathrm{~cm}^{-1-3}$ is attributable to $C-C$ stretching vibrations from the polymer.

\section{General Discussion}

\section{Polymer Monolayer}

The current study investigates the effect of a polycation on calcium phosphate mineralization at the air-water interface. At the air-water interface, P1 (Figure 1) exhibits a behavior reminiscent of poly(acrylic acid)-block-poly(n-butylacrylate) (PAA-b-PnBA) block copolymers. ${ }^{[25]}$ At $\mathrm{pH} 2$, the polymer shows no specific features and only a gas to liquid phase transition before the collapse pressure of $60 \mathrm{mN} \cdot \mathrm{m}^{-1}$. At $\mathrm{pH} 5$ and higher, a transition from gas to expanded liquid can be observed.

These observations are consistent with Rehfeldt et al., ${ }^{[36]}$ who reported monolayer formation of poly(methyl methacrylate)-block-poly(dimethylethylamino methacrylate) (PMMA- $b$-PDMAEMA) at the air-water interface. Essentially, their $\pi-A$ isotherms are identical to our isotherms, but the authors did not report on the collapse pressure and the specifics of the observed phase transition(s). They have, however, shown that lowering the $\mathrm{pH}$ value of the subphase leads to a dramatic decrease in the mean molecular area of their polymers. This is because of protonation of the basic groups in the PDMAEMA block, similar to the current data. As the polymer used by Rehfeldt et al. is much more symmetric and less hydrophilic than P1 (PMMA ${ }_{36}{ }^{-b}$ PDMAEMA $_{36}$ vs. PnBMA ${ }_{75}$-PDMAEMA ${ }_{147}$ ) it is possible that polymer loss to the subphase, especially at low $\mathrm{pH}$, is more pronounced in the current study.

AFM experiments on transferred polymer films (Figure 2) confirm that the films are stable, uniform, nearly defect-free, and smooth with a roughness below $0.2 \mathrm{~nm}$ on hydrophilic surfaces. In contrast, films on hydrophobic surfaces show a high degree of dewetting. We assign this behavior to the fact that: 1) the polymer is rather large and it may, therefore, not be transferred in an equilibrium geometry and 2) the hydrophilic block is about two thirds of the entire polymer and it may, therefore, not be straightforward 

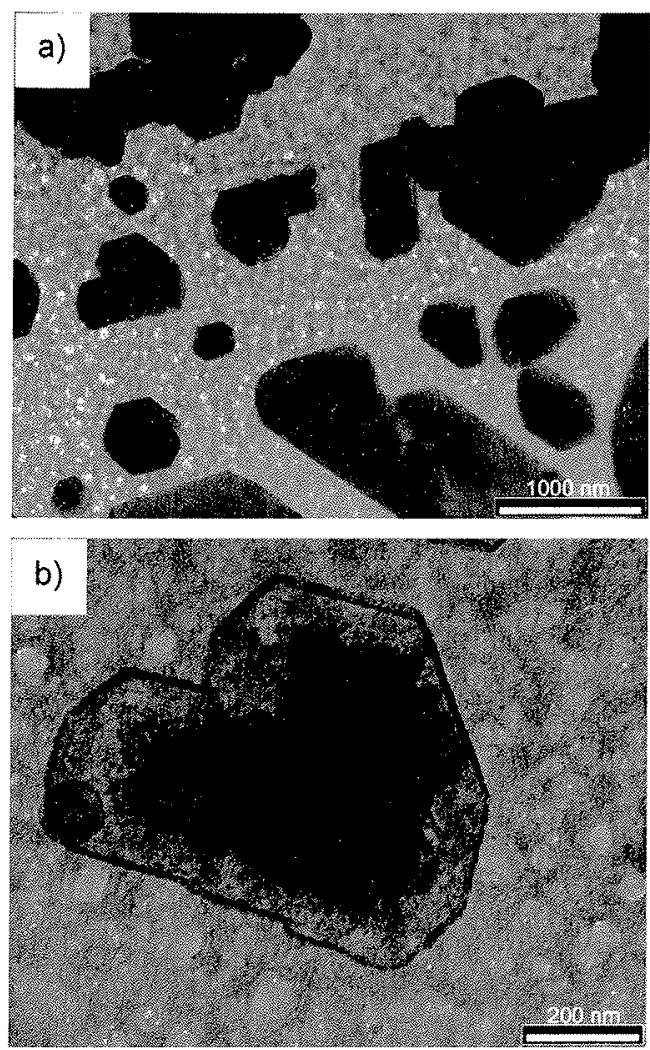

Figure 4. TEM images of mineralization products obtained at $\mathrm{pH} 5$ after $12 \mathrm{~h}$ without stirring. The bright edge region in the crystals in panel (b) is a result of beam damage.

to transfer a dense film onto a hydrophobic substrate at $30 \mathrm{mN} \cdot \mathrm{m}^{-1}$

In contrast to the above observations, Casse et al. ${ }^{[25]}$ have shown that the $\mathrm{pH}$ and the ionic strength have little effect on the stability of PAA-b-PnBA monolayers at the air-water interface. These authors have demonstrated that in the case

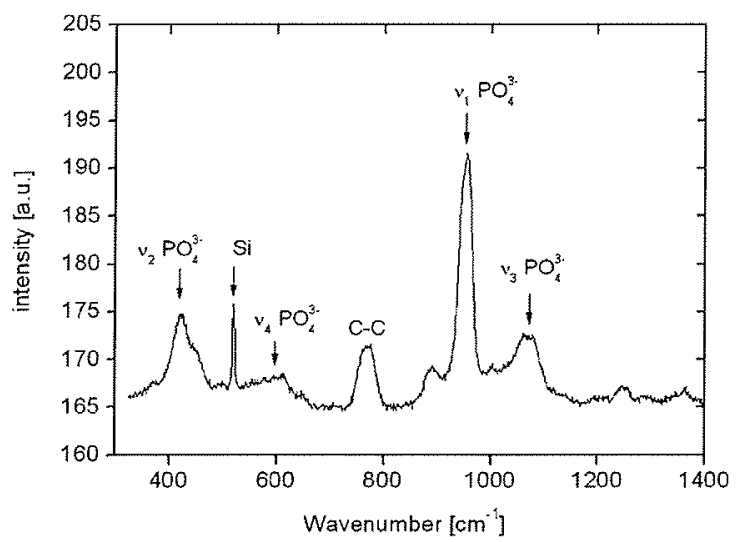

Figure 5. Raman spectrum of $\mathrm{P}_{1}$ mineralized with calcium phosphate at $\mathrm{pH} 8$. of PAA- $b$-PnBA, the hydrophobic block is responsible for the monolayer stability. The current work and two earlier studies $^{[25,36]}$ thus suggest that polycationic and polyanionic block copolymer monolayers show significant differences with respect to their behavior, although more data is needed for a final conclusion.

\section{Monolayer Mineralization}

Raman (Figure 5) and IR spectroscopy along with EDXS (Figure 3f) of the mineralized films confirm the formation of calcium phosphate. In contrast to earlier studies, ${ }^{[3,34]}$ the crystal phase of the precipitate can (at least at high pH, HAP) be identified. In agreement with the well known variation of the crystal phase with the reaction conditions, ${ }^{[4]}$ AFM (Figure 2c) shows that the mineralization at $\mathrm{pH} 5$ leads to uniform, almost defect-free films with rounded features between 20 and $50 \mathrm{~nm}$. More interestingly, TEM (Figure 3) shows that the $\mathrm{pH}$ has a strong influence on the morphology of the calcium phosphate precipitates. At $\mathrm{pH} \mathrm{5,} \mathrm{uniform}$ objects connected by filaments with a diameter of 2 to $3 \mathrm{~nm}$ form. These precipitates are similar to calcium phosphate grown by Peytcheva et al. ${ }^{[33]}$ and Antonietti et al., ${ }^{[34]}$ although much smaller. This is possibly a result of the preparation procedures, which are different (bulk vs monolayer mineralization). In our case, nucleation and growth only occurs on a 2D interface, while in the earlier studies nucleation and growth can occur within the bulk phase. Moreover, the two earlier studies used much higher calcium and phosphate concentrations $(0.5$ and $0.3 \mathrm{M}$, respectively vs $2 \times 10^{-3} \mathrm{M}$ in the current study), which complicates a final conclusion.

At pH 8, much larger and less well defined particles are observed. They are composed of smaller elongated features, which are in turn composed of thin fibrillar objects. They are (within one of the elongated features) approximately parallel to one another, while the fllaments obtained at $\mathrm{pH} 5$ are not oriented. The orientation within one of the features is not a transfer artifact, because the features themselves are not oriented. Moreover, these structures are similar to precipitates observed by other groups. Li et al. investigated the precipitation of hydroxyapatite in the presence of different Pluronics triblock copolymers at $\mathrm{pH} 10$ and showed that the precipitates consist of calcium phosphate nanofibers. ${ }^{[37]}$ Bigi et al. found similar objects in the presence of low-molecular-weight poly(acrylic acid) and different concentrations of magnesium and fluoride ions. ${ }^{[38]}$ Neither study however, commented on the reason for the formation of such structures.

The current study thus shows that positively charged polymer monolayers at the air-water interface have a strong effect on calcium phosphate mineralization. These effects are distinctly different from the effects of analogous, but negatively charged, polymer monolayers. In the latter case, ${ }^{[25]}$ the most highly ordered mineral films are obtained 
at high pH, where the PAA block is deprotonated and highly charged. In the current study, the most well structured mineral films were obtained at $\mathrm{pH}$ values around 5 , where the PDMAEMA block $\left(\mathrm{p} K_{\mathrm{a}}=7.5\right)^{[39]}$ is protonated. This suggests that in both cases, the polymer must be charged for uniform nucleation and growth.

We thus propose, similar to Zahn and coworkers (for another chemical system, gelatin) ${ }^{[40]}$ and Shkilnyy et al. (for polyethyleneimine hydrogels), ${ }^{[27]}$ that the first interaction of the charged polymer monolayer with the incoming inorganic ions is crucial for the nucleation and growth process. Presumably, the charged polymer surfaces act as a medium for the enrichment of the inorganic ions (e.g., phosphate) close to the surface, which leads to a locally high ion concentration in the polymer brush. Upon addition of the second inorganic ion, e.g., calcium, the preferred nucleation site is within the polymer monolayer, which leads to complex morphologies like the ones observed in the TEM images. The resulting hybrid film is relatively rich in organic material (as demonstrated by EDXS, Figure 3, Raman, Figure 5, and IR, not shown), which confirms an intimate association of the inorganic with the polymer monolayer. A phosphate-induced assembly has indeed also been proposed for polycation-mediated silica formation and calcium phosphate formation in the presence of poly(t-lysine) block copolymers. ${ }^{[28,40]}$

Filaments such as those just discussed have been observed before. Interestingly, however, they have been made using a polycarboxylate at $\mathrm{pH} 5 \cdot \cdot^{[33,34]}$ The experimental approach in these studies was quite different from the current work (double-jet precipitation vs mineralization of a polymer monolayer, different concentrations and reaction times), so it is at the moment difficult to draw a final conclusion. One possible explanation for the observations made in our study could, however, be an interaction between the phosphate ions and the polycation, which leads to a pre-organization of the polymer matrix. Such an effect has been observed in silica biomineralization, ${ }^{[40]}$ but remains to be proven for systems such as the one presented here.

Finally, stirring has, similar to an earlier study, ${ }^{[25]}$ a strong effect on the morphology of the calcium phosphate. Without stirring, a dense network of fiber-like entities forms. This network carries much larger, plate-like particles (Figure 4). These particles have relatively well-defined crystal faces and appear to be in register with the neighboring large crystals. While this may suggest a long-range correlation, it is most likely a transfer artifact involving loosely bound large crystals, which are immobilized in an oriented manner after transfer and drying of the film.

In summary, the study shows that calcium phosphate formation beneath polycationic monolayers is significantly different from calcium phosphate formation on polymer multilayers on solid substrates. ${ }^{[18,20-22]}$ Earlier studies either show the formation of small, rather dense particles or very large fluffy aggregates. Occasionally, the particle shape is only roughly described. Moreover, the crystal phases have only rarely been identified. This is mostly because there is simply not enough material for a complete phase analysis and most authors must, therefore, rely on IR spectroscopy, which can be misleading.

The morphologies observed in the current study differ in several respects from earlier work. 1) The particle morphologies observed in the current study at $\mathrm{pH} 8$ are much coarser than the morphology observed by Ball et al. ${ }^{[20]}$ and much less homogeneous than results by Ngankam et al. ${ }^{[18]}$ 2) The only study reporting on the effects of cationic surfactants at the air-water interface on calcium phosphate formation shows that small features with sizes similar to those observed here form. ${ }^{[24]}$ However, the TEM data in this publication are not very clear and it is thus difficult to decide whether or not there is a real difference between a cationic surfactant layer and a polycationic polymer layer on calcium phosphate mineralization. Unfortunately, these authors have not reported on the mineralization at lower $\mathrm{pH}$, which makes a comparison with our work difficult.

The unique feature of the current study is indeed that the effect of polycations at the air-water interface is for the first time evaluated at different $\mathrm{pH}$ values, including nonphysiological conditions. Open questions, therefore, concern the role of the $\mathrm{pH}$ on calcium phosphate mineralization on polymer films such as those just discussed. ${ }^{[18,20-22,24]}$ Unfortunately there are no data available on this topic at the moment, yet our work suggests that the degree of charging, also in polycations, is a key influence in calcium phosphate formation.

\section{Conclusion}

Calcium phosphate is an important, but complex biomineral, and its formation and structure-property relations at biological and biomimetic conditions are still a matter of debate. A key finding of the current study is that polycationic interfaces are useful model systems to better understand calcium phosphate mineralization under biomimetic conditions. Consistent with earlier studies, the most well defined calcium phosphate films form if the polymer is charged. This enables (by electrostatic interaction) an efficient enrichment of the precursor ions within the polymer, which in turn is an important requirement for controlled nucleation, growth, and organization of the calcium phosphate precipitates. At low $\mathrm{pH}$, polycations such as PDMEAMA are very efficient, because they are highly charged. Possibly, there is an additional effect, the pre-organization of the polycationic matrix by the 
phosphate ions, which could be a key factor in the formation of the intriguing filaments observed in the TEM.

Acknowledgements: The authors thank B. Stiller for AFM measurements, E. Bieler for help with SEM and TEM measurements, Dr. A. Masic for access to the Raman microscope, and the MPI of Colloids and Interfaces (Colloid Chemistry Department), the University of Potsdam, the German Research Foundation, and the Swiss National Science Foundation for financial support. M.J. acknowledges a doctoral fellowship from the MPI-KG.

Keywords: biomimetic; biomineralization; calcium phosphate; polymer monolayer

[1] P. Behrens, E. Baeuerlein, Handbook of Biomineralization, Vol 2. Wiley-VCH, Weinheim 2007.

[2] K. Gorna, R. Munoz-Espi, F. Gröhn, G. Wegner, Macromol. Biosci. 2007, 7, 163.

[3] H. Cölfen, S. Mann, Angew. Chem., Int. Ed. 2003, 42, 2350.

[4] S. Schweizer, A. Taubert, Macromol. Biosci. 2007, 7, 1085.

[5] F. Variola, F. Vetrone, L. Richert, P. Jedrzejowski, J. H. Yi, S. Zalzal, S. Clair, A. Sarkissian, D. F. Perepichka, J. D. Wuest, F. Rosei, A. Nanci, Small 2009, 5, 996.

[6] N. A. J. M. Sommerdijk, G. de With, Chem. Rev. 2008, 108, 4499.

[7] F. C. Meldrum, H. Cölfen, Chem. Rev. 2008, 108, 4332.

[8] R. Q. Song, H. Cölfen, A. W. Xu, J. Hartmann, M. Antonietti, ACS Nano 2009, 3, 1966.

[9] A. W. Xu, W. F. Dong, M. Antonietti, H. Cölfen, Adv. Funct. Mater. 2008, 18, 1307.

[10] H. Cölfen, Biomineralization II: Mineralization Using Synthetic Polymers and Templates, Vol. 271, Springer, Berlin, 2007, p. 1.

[11] T. Y. J. Han, J. Aizenberg, Chem. Mater. 2008, 20, 1064.

[12] Y. Politi, D. R. Batchelor, P. Zaslansky, B. F. Chmelka, J. C. Weaver, I. Sagi, S. Weiner, L. Addadi, Chem. Mater. 2009, 22, 161.

[13] F. F. Amos, D. M. Sharbaugh, D. R. Talham, L. B. Gower, M. Fricke, D. Volkmer, Langmuir 2007, 23, 1988.

[14] E. DiMasi, V. M. Patel, M. Sivakumar, M. J. Olszta, Y. P. Yang, L. B. Gower, Langmuir 2002, 18, 8902 .

[15] E. DiMasi, S. Y. Kwak, F. F. Amos, M. J. Olszta, D. Lush, L. B. Gower, Phys. Rev. Lett. 2006, 97.

[16] L. Addadi, D. Joester, F. Nudelman, S. Weiner, Chem. Eur. J. 2006, 12, 981.
[17] F. Nudelman, E. Shimoni, E. Klein, M. Rousseau, X. Bourrat, E. Lopez, L. Addadi, S. Weiner, J. Struc. Biol. 2008, 162, 290.

[18] P. A. Ngankam, P. Lavalle, J. C. Voegel, L. Szyk, G. Decher, P. Schaaf, F. J. G. Cuisinier, J. Am. Chem. Soc. 2000, 122, 8998.

[19] D. Volkmer, M. Fricke, C. Agena, J. Mattay, J. Mater. Chem. 2004, 14, 2249.

[20] V. Ball, M. Michel, F. Boulmedais, J. Hemmerle, Y. Haikel, P. Schaaf, J. C. Voegel, Cryst. Growth Des. 2006, 6, 327.

[21] E. D. Spoerke, S. I. Stupp, Biomaterials 2005, 26, 5120.

[22] S. Schweizer, T. Schuster, $M$. Junginger, G. Siekmeyer, A. Taubert, Macromol. Mater. Eng. 2010, 10.1002/ mame200900347.

[23] D. Walsh, E. Boanini, J. Tanaka, S. Mann, J. Mater. Chem. 2005 $15,1043$.

[24] L.-J. Zhang, H.-G. Liu, X.-S. Feng, R.-J. Zhang, L. Zhang, Y.-D. Mu, J.-C. Hao, D.-J. Qian, Y.-F. Lou, Langmuir 2004, 20, 2243.

[25] O. Casse, O. Colombani, K. Kita-Tokarczyk, A. H. E. Müller, W. Meier, A. Taubert, Faraday Discuss. 2008, 139, 179.

[26] A. Shkilnyy, A. Friedrich, B. Tiersch, S. Schöne, M. Fechner, J. Kötz, C. W. Schlapfer, A. Taubert, Langmuir 2008, 24, 2102.

[27] A. Shkilnyy, R. Graf, B. Hiebl, A. T. Neffe, A. Friedrich, J. Hartmann, A. Taubert, Macromol. Biosci. 2009, 9, 179.

[28] A. Shkilnyy, J. Brandt, A. Mantion, O. Paris, H. Schlaad, A. Taubert, Chem. Mater. 2009, 21, 1572.

[29] It must be noted that the stability of the polymer film was monitored at constant surface pressure and not at constant mean molecular area, because we intended to maintain the mineralization conditions as uniform as possible.

[30] M. P. Ginebra, E. Fernandez, E. A. P. DeMaeyer, R. M. H. Verbeeck, M. G. Boltong, J. Ginebra, F. C. M. Driessens, J. A. Planell, J. Dental Res. 1997, 76, 905.

[31] J. H. Bradt, M. Mertig, A. Teresiak, W. Pompe, Chem. Mater. 1999, 11, 2694

[32] G. He, T. Dahl, A. Veis, A. George, Nat. Mater. 2003, 2, 552.

[33] A. Peytcheva, H. Cölfen, H. Schnablegger, M. Antonietti, Colloid Polym. Sci. 2002, 280, 218.

[34] M. Antonietti, M. Breulmann, C. G. Göltner, H. Cölfen, K. K. W. Wong, D. Walsh, S. Mann, Chem. Eur. J. 1998, 4, 2493.

[35] M. Lazzeri, F. Mauri, Phys. Rev. Lett. 2003, 90.

[36] F. Rehfeldt, R. Steitz, S. P. Armes, R. von Klitzing, A. P. Gast, M. Tanaka, J. Phys. Chem. B 2006, 110, 9171.

[37] Y. B. Li, D. X. Li, Z. Z. Xu, J. Mater. Sci. 2009, 44, 1258.

[38] E. Bertoni, A. Bigi, G. Cojazzi, M. Gandolfi, S. Panzavolta, N. Roveri, J. Inorg. Biochem. 1998, 72, 29.

[39] P. van de Wetering, E. E. Moret, N. M. E. Schuurmans-Nieuwenbroek, M. J. van Steenbergen, W. E. Hennink, Bioconjugate Chem. 1999, 10, 589.

[40] K. Lutz, C. Groger, M. Sumper, E. Brunner, Phys. Chem. Chem. Phys. 2005, 7, 2812. 